\title{
LA INFLORESCENCIA EN LAS ESPECIES DEL GÉNERO TEUCRIUM L. (LAMIACEAE) PRESENTES EN LA PENÍNSULA IBÉRICA Y BALEARES
}

\author{
Teresa NAVARRO y Baltasar CABEZUDO
}

\begin{abstract}
RESUMEN. La inflorescencia en las especies del género Teucrium L. (Lamiaceae) presentes en la Península Ibérica y Baleares. En las especies ibéricas del género Teucrium, se detectan dos tipos de estructuras básicas inflorenciales; el tirso indeterminado de verticilastros (secciones Teucrium, Scorodonia, Spinularia, Scordium y Chamaedrys) y el racimo condensado "glomérulos» (o cabezuelas) (sección Polium). Las inflorescencias racemosas indeterminadas y politélicas son derivadas del tirso. Los tipos de ramificación en infloresecencias compuestas, racemosas, indeterminadas, politélicas y heterotéticas son: eje principal no ramificado terminado en racimo, doble racimo y racimo de doble racimo. Se han observado grados de transformaciones evolutivas por procesos de racemización, truncación, condensación floral y floración supernumeraria que son más frecuentes en especies cultivadas que en especies desarrolladas en el medio natural. La variación que pueden experimentar las inflorescencias de una misma especie son debidas a transformaciones evolutivas por adaptación a condiciones ecológicas específicas.
\end{abstract}

Palabras clave. Inflorescencia, Teucrium L., Península Ibérica, Islas Baleares, España.

ABSTRACT. The inflorescence in the species of the Teucrium L., genus in the Iberian peninsula and Baleares the Balearic Islands. The inflorescences of the genus Teucrium L., in the Iberian peninsula have been studied. Two main groups (florescence) are described: indeterminate thyrse with the partial florescence and condensed raceme. The types of branching in compound polytelic and heterothetic racemose inflorescence are: infloresecence axis terminates with a single condensed raceme; double raceme and raceme of double raceme. The evolutionary transformations by the processes of racemization, truncation, condensation and superflowering are detected. The cultivated plants showed higher evolutionary transformations. The most variations in the inflorescence type are the specific environmental adaptations.

Key words. Inflorescence, Teucrium L., Iberian peninsula, Balearic Islands, Spain.

\section{INTRODUCCIÓN}

La inflorescencia es un carácter sistemático de interés en el género Teucrium L. (Willkomm, 1868; Kästner, 1978,1989; Puech, 1984; Bayón, 1990; Mus et al., 1991; El Oualidi \& Puech, 1993; Navarro, 1995), la variabilidad estructural y tipológica expresa el polimorfismo que caracteriza al género y la respuesta adaptativa al estrés ambiental.

El objetivo de este trabajo es definir aspectos básicos de la morfologia floral en el género Teucrium L. (Península Ibérica e Islas Baleares), caracterizar modelos florales y 
describir la presencia de diferentes grados de inflorescencias derivadas por transformaciones evolutivas originadas por racemización, truncación, condensación floral y floración supernumeraria, y su relación con los fenómenos de adaptación. Para ello se han seguido los trabajos de Ricket (1944, 1955), Troll (1950, 1964, 1969), Maresquelle (1961, 1970), Webberling (1963, 1989), Sell (1964, 1969), Hutchinson (1969), Meena Sharma \& V. Singh (1980), y Kunze (1989).

\section{MATERIAL Y MÉTODOS}

Las observaciones se realizaron en material vivo (campo y cultivo) y en material seco conservado en los herbarios MGC, GDAC, SEV, MA, ALME, BC y MUB. Las inflorescencias de material de hebario examinadas se rehidrataron previamente. En los taxones con amplia distribución se estudiaron poblaciones de toda su área, para detectar variabilidad por adaptación a hábitats específicos.

\section{RESULTADOS}

\section{Estructura y organización general}

La inflorecencia, en el género Teucrium, es fundamentalmente racemosa, indeterminada, politélica y heterotética (Troll, 1964). En las especies donde la inflorescencia se ramifica (sinflorescencia en el sentido de Troll), una florescencia principal queda en la zona distal del eje principal (florescencia), debajo de ella se disponen ramas florales (paracladios 0 ramificaciones parciales) las cuales repiten la organización morfológica de la florescencia terminal y se llaman coflorescencias (Troll, 1964) (fig. 1). En los casos donde la inflorescencia no se ramifica, solo la florescencia principal está presente (fig. 2A)
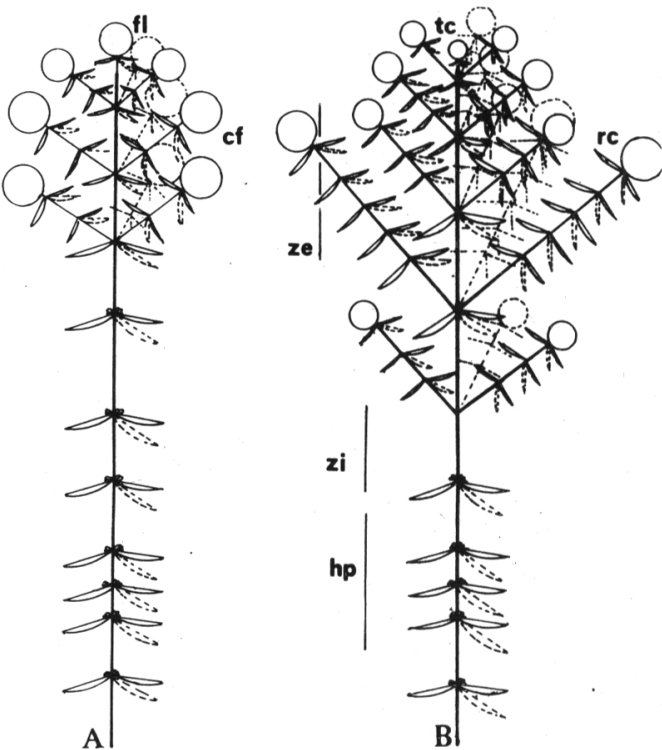

Figura 1. Estructura inflorencial: A.- Estructura inflorencial de un racimo de racimos no afectada por transformaciones evolutivas. B.- la misma afectada por fenómenos de truncación (tc) y racemización (rc). (fl) (florescencia, inflorescencia principal); (cf) (coflorescencia, ramificaciones parciales o paracladios); (ze) (zona de enriquecimiento); (zi) (zona de inhibición); (hp) (hipotagma). Los círculos en blanco corresponden a glomérulos o racimos condensados. Inflorential structure. A.- Inflorential structure of a double raceme not affected by evolutionary transformations. B.- affected by truncation processes (tc) and racemization $(r c)$. ( $f l)$ (florescence, main inflorescence); (cf) (coflorescence, partial branches or paraclades); (ze) (enrichment zone); (zi) (inhibition zone); ( $h p$ ) (hypotagma). The white circle is a condensed raceme.

Las flores se organizan en dos tipos de inflorescencias principales (florescencia), que definen dos estructuras florales básicas: tirso de verticilastros y racimos condensados (glomérulos o cabezuelas).

El tirso de verticilastros, inflorescencia tírsica (Briggs \& Johnson, 1979) deriva de un tirso de inflorescencias parciales cimosas (Troll, 1964), las modificaciones de estas cimas 
o dicasios, por acortamiento de los pedicelos florales, reducción o enriquecimiento floral, originan verticilastros dispuestos espaciadamente a lo largo del eje principal, con flores ligeramente pediceladas y de inserción unilateral.

El racimos condensado deriva del tirso de verticilastros por reducción floral en cada uno de ellos, generalmente por aborto de las dos flores laterales del dicasio, originando verticilastros bifloros, que se contractan o agrupan en «glomérulos», «cabezuelas»o "espigastros». Estos forman ejes cortos con (6) 12-25(45) flores sesiles o subsesiles de inserción helicoidal, donde las flores apicales abortan conforme aumenta su número. Hay géneros de labiadas como Satureja, Phlomis, Ballota, Salvia y Sideritis con inflorescencia en tirso de verticilastros y otros como Origanum, Thymus, Thymbra, Mentha y Lavandula con inflorescencia racemosa en racimo condensado. La presencia de ambos
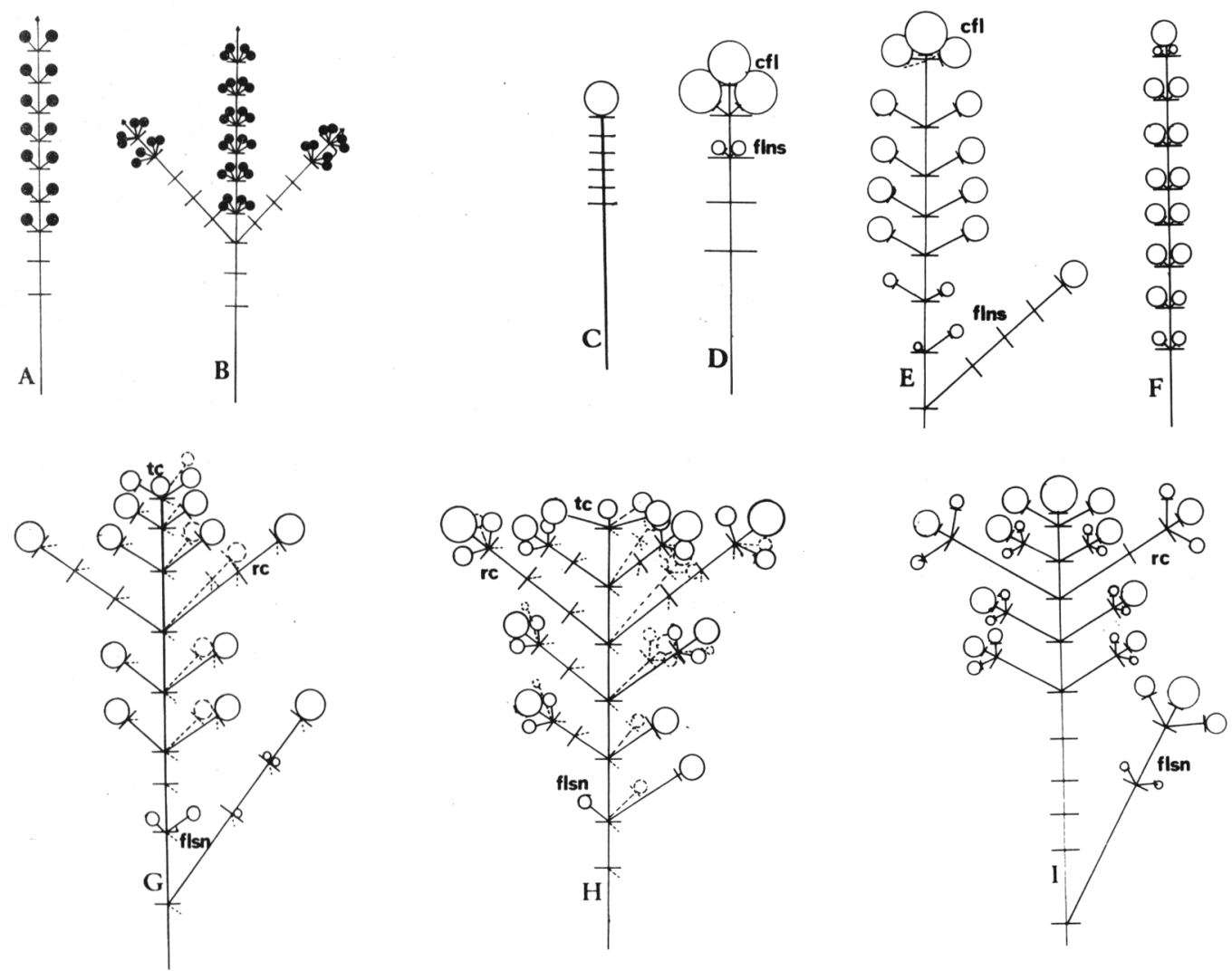

Figura 2. Variabilidad tipológica: A.- tirso simple de verticilastros bifloros. B.- tirso ramificado de verticilastros de 4 flores. C.- racimo condensado terminal (glomérulo). D.- racimo corto de racimos. E, F, G.- racimo de racimos (doble racimo). I, H.- racimo de dobles racimos. E, I con ramificación parcial opuesta; G, H con ramificación parcial verticilada. (flsn) floración supernumeraria; (cfl) condensación floral; (rc) racemización; (tc) débil truncación. Los círculos en blanco corresponden a glomérulos; los negros a una flor simple. Tipological variability: A. simple bifloral verticillated thyrse. B.- ramificated four flowered verticillated thyrse. C.-condensed raceme. E, F, G.-double raceme. I, H. - raceme of double raceme. E, I.- with partial and opposite branches. G, H.- with partial and verticillated branches. (flsn) superflowering; (cfl) floral condensation; $(r c)$ racemization; $(t c)$ weak truncation. The black circle is a single flower, the white circle is a condensed raceme. 
tipos en un mismo género, caso de Teucrium y Thymus, es menos frecuente.

En todas las secciones estudiadas del género, a excepción de la sección Polium, la inflorescencia principal es el tirso de verticilastros, con número de flores por verticilastro variable (2)4-6(8) siendo un carácter que permite diferenciar especies. La inflorescencia se forma en ramas de un año y generalmente en la misma estación que las ramas vegetativas.

En la sección Polium, la inflorescencia principal es siempre un racimo condensado, la variación en la forma (cabezuela o espiga), densidad floral con (4)6-12(45) flores y tamaño $(0,5) 1-15(20) \mathrm{cm}$, son caracteres que sirven para diferenciar especies. La inflorescencia se origina generalmente en ramas de más de un año y en diferente estación que la vegetativa.

\section{Variabilidad tipológica}

La inflorescencia en tirso de verticilastros, puede ser simple o ramificada (fig. 2A, B). La ramificación opuesta tiene lugar en la zona proximal del eje principal o rara vez en la distal (Mus et al., 1991) y repite el modelo de la inflorescencia principal. La estructura inflorencial es siempre la misma, siendo su variabilidad tipológica escasa, la variación depende si existe o no una modificación morfológica de las bracteas respecto a las hojas caulinares, del número de flores por verticilastro (1)2-(4)8 y del número de verticilastros por inflorescencia (5)8-(10)25 (secciones Teucrium, Chamaedrys, Scordium, Spinularia y Scorodonia)

En las inflorescencias en racimo condensado (glomérulos, espigastros 0 cabezuelas), la variabilidad tipológica es mayor que en el caso anterior, las brácteas pueden ser semejantes morfológicamente a las hojas caulinares o no, y el tipo de ramificación parcial es opuesta o verticilada con 3-6 ramas. Los niveles de organización observados segun el tipo de ramificación son: a) Racimo condensado terminal (fig. 2C), situado en el extremo del eje principal no ramificado (Weberling, 1989), pudiendo ser laxifloro (subsección Rotundifolia o densifloro (el resto de las subsecciones); b) Racimo de racimos (doble racimo), las ramificaciones parciales pueden ser opuestas (fig. 2E, F) o verticiladas (fig. $2 \mathrm{G}$ ) el número de orden de ramificación varía de 6 a 12, y el de glomérulos de (10)12-16(35), su estructura sin ser una verdadera panícula, presenta en ocasiones un aspecto semejante a ella (Troll, 1964). c) Racimo corto de racimos (fig. 2D), cuando en el doble racimo el número de orden de ramificaciones parciales es bajo (1 ó 2) y el número de glomérulos de 3 a 5. d) Racimo de dobles racimos (fig. $2 \mathrm{H}, \mathrm{I}$ ), cuando las ramificaciones parciales se enriquecen y repiten la estructura básica de un doble racimo (florescencia). (Anexo).

\section{Transformaciones evolutivas}

Se han observado fenómenos (Werberling, 1989) que constituyen diferentes grados de transformaciones evolutivas en inflorescencias politélicas. Estos fenómenos son frecuentes en familias como Acanthaceas (Sell, 1969), Mirtaceas, Leguminosas y Compuestas (Kunze, 1989) e incrementan la complejidad de la estructura inflorencial. Básicamente los fenómenos observados en algunas especies del género Teucrium son: a) Racemización (fig. $2 \mathrm{G}, \mathrm{H}, \mathrm{I})$ cuando el número de inflorescencias parciales aumenta por enriquecimiento, las ramificaciones parciales de la zona distal se racemizan de manera que se tiende a una inversión de la floración primitivamente basípeta a acrópeta. Este fenomeno se acompaña de una reducción de la longitud y tamaño de las ramificaciones parciales situadas inmediatamente debajo de la inflorescencia principal además de una tendencia a la reducción floral de la misma; b) Truncación 
(fig. 2G, H, I) cuando la racemización se acentúa, la inflorescencia principal pierde dominancia apical y se estimula el desarrollo de las ramificaciones parciales, que tienden a sustituir a la principal; c) Floración supernumeraria (fig. 2E, G, H) es una floración de refuerzo suplementaria que aparece bajo forma de flores, yemas preflorales 0 inflorescencias axilares que no llegan a convertirse en verdaderas inflorescencias parciales y d) condensación floral (fig. 2E, G) es la reagrupación de las inflorescencias parciales en la zona distal del eje floral, acompañada de una reducción de la distancia internodal.

Las transformaciones evolutivas han sido observadas fundamentalmente en la sección Polium y más raramente en el resto, donde solo se detectan en especies sufrutescentes con tirsos de más de 10 verticilastros. Las ramificaciones parciales del eje principal que presentan tendencia a la racemización son las localizadas en el 3 ó 4 orden de ramificación bajo la inflorescencia principal, y las que tienden a sustituir la inflorescencia principal en el caso de tendencia a truncarse corresponden a las situadas en el 2 ó 3 orden. Ambos procesos, racemización y ligera truncación se detectan con diferentes grados en $T$. lusitanicum, $T$. capitatum, T. haenseleri, T. eriocephalum, $T$. turredanum y $T$. dunense. La floración supernumeraria nunca es homogenea y se expresa por la presencia de yemas preinflorenciales que abortan o por cortas ramificaciones parciales en la zona proximal del eje principal (hipotagma), es más frecuente en especies en cultivo que en el medio natural y se observa en $T$. lusitanicum, T. capitatum, $T$. murcicum, T. haenseleri, $T$. similatum, $T$. dunense. El fenomeno de condensación floral está asociado a zonas con ecotonía o estrés y se caracteriza por inflorescencias «congestas» 0 contractadas en el extremo distal del eje principal, aparecen en; $T$. dunense, $T$. charidemi, T. similatum, $T$. aragonense, $T$. angustissimum, T. murcicum, T. capitatum y $T$. lusitanicum. (Anexo).

\section{ANEXO}

Tipología inflorencial de las especies del género Teucrium en la Península Ibérica y Baleares. Las especies en negrita pueden presentar fenómenos de transformaciones evolutivas

Inflorescence typology of the species Teucrium genus in the Iberian peninsula and Balearic islands. The species in black print may present the evolutionary transformation processes.

\section{Tirso de verticilastros}

$$
\begin{aligned}
& \text { Verticilastros de } \mathbf{1 - 2} \text { flores } \\
& \text { Sect. Spinularia } \\
& \text { T. spinosum L. } \\
& \text { Verticilastros de } 2 \text { flores } \\
& \text { Sect. Teucrium } \\
& \text { T. fruticans } \mathrm{L} \text {. } \\
& \text { T. aristatum Pérez Lara } \\
& \text { T. campanulatum L. } \\
& \text { T. pseudochamaepitys L. } \\
& \text { Sect. Scordium } \\
& \text { T. botrys } \mathrm{L} . \\
& \text { T. resupinatum } \text { Desf. } \\
& \text { Sect. Chamaedrys } \\
& \text { T. intrincatum Lange } \\
& \text { Sect. Scorodonia } \\
& \text { T. scorodonia } \mathrm{L} \text {. } \\
& \text { T. oxylepis } \text { Font Quer } \\
& \text { T. asiaticum } \mathrm{L} \text {. } \\
& \text { T. salviastrum } \text { Schreb. }
\end{aligned}
$$

Verticilastros de 2-4 flores

Sect. Chamaedrys

T. marum L.

Verticilastros de 2-6 flores

Sect. Chamaedrys

T. chamaedrys L.

T. fragile Boiss.

T. webbianum Boiss.

Verticilastros de 2-8 flores

Sect. Scordium

T. scordium L.

\section{Racimo condensado}

Racimo condensado terminal

Sect. Polium Subsect. Polium

T. angustissimum Schreb.

T. aragonense Loscos \& J. Pardo

T. carthaginense Lange

$T$. cossonii Wodd

T. edetanum M. B. Crespo, Mateo \& T. Navarro

T. expanssum Pau

T. gnaphalodes L'Hér. 
T. homotrichum (Font Quer) Rivas Mart.

T. leonis Sennen

T. lerrouxi Sennen

T. luteum (Mill.) Degen

T. polium L.

T. similatum T. Navarro \& Rosúa

T. turdetanum (Devesa \& Valdes. Berm.) Peris

$\& a l$

$T$. vincentinum Rouy

Sect. Polium Subsect. Pumilum

T. balthasaris Sennen

T. carolipaui Vicioso ex Pau

T. lepicephalum $\mathrm{Pau}$

T. libanitis Schreb.

T. pumilum L.

Sect. Polium Subsect. Rotundifolia

T. buxifolium Schreb.

T. cavanillesianum Font Quer \& Jerónimo

T. compactum Clemente ex Lag.

T. chrysotrichum Lange

T. franchetianum Rouy ex Coincy

T. freynii Willk.

T. hifacense Sennen

T. montanum $\mathrm{L}$.

T. pyrenaicum $\mathrm{L}$.

T. rivas-martinezii Alcaraz \&al.

T. rivasii Rigual

T. rixanense Ruíz Torre \& Ruíz del Cast.

T. rotundifolium Schreb.

T. thymifolium Schreb.

Sect. PoliumSubsect. Simplicipilosa

T. lanigerum Lag.

Racimo corto de racimos

Sect. Polium subsect. Polium

T. aragonense Loscos \& J. Pardo

$T$. carthaginense Lange

T. cossonii Wood

T. expanssum Pau

$T$. gnaphalodes L'Hér.

T. luteum (Mill.) Degen

T. murcicum Sennen

T. polium L.

T. similatum $\mathrm{T}$. Navarro \& Rosúa

Sect. Polium Subsect. Simplicipilosa

T. lanigerum Lag.

Doble racimo

Sect. Polium Subsect. Polium

T. algarbiense (Cout.) Cout.

T. capitatum $\mathrm{L}$.

T. dunense Sennen

$T$. hieronymi Sennen

T. lusitanicum Schreb.

T. murcicum Sennen

T. polium L.

T. reverchonii Willk.

Sect. Polium Subsect. Simplicipilosa

T. charidemi Sandwiht
T. almeriense C.E. Hubb. \& Sandwiht

T. eriocephalum Willk.

T. haenseleri Boiss.

$T$. lanigerum Lag.

Racimo de dobles racimos

Sect. Polium Subsect. Polium

T. capitatum $\mathrm{L}$.

T. hieronymi Sennen

T. lusitanicum Schreb.

Sect. Polium Subsect. Simplicipilosa

T. almeriense C. E. Hubb. \& Sandwiht

T. eriocephalum Willk.

T. haenseleri Boiss.

Sect. Polium Subsect. Pumilum

T. turredanum Losa \& Rivas Goday

\section{BIBLIOGRAFÍA}

BAYÓN ALVARO, E. -1990- Revisión taxonómica del género Teucrium L. (Labiatae) (Excl. Sect. Polium (Mill.) Schreb. en la Península Ibérica e Islas Baleares. 251 pp. Universidad de Leon.

BRIGGS, B.G. \& L.A.S. JOHNSON. -1979Evolution in the Myrtaceae evidence from inflorescence structure. Proc. Linn. Soc. New South Wales, 102: 157-256.

EL OUALIDI, J. -1991-Biosystematique et taxinomie des Teucrium de la Section Polium (Lamiaceae) dans le bassin méditerranéen occidental. Thése de Doctorat. Université de Montpellier (Montpellier II). Sciences et Techniques du Languedoc. $219 \mathrm{pp}$.

EL OUALIDI, J \& S, PUECH -1993- Quelques marqueurs morphologiques des Teucrium section Polium (Lamiaceae) du Maroc. Valeurs diagnostiques à differents niveaux d'integration. Acta Bot. Malacitana, 18: 163-173.

HUTCHINSON, J. -1969. Evolution and phylogeny of flowering plant. Academic press Ltd. London.

KÄSTNER, A. -1978- Beiträge zur wuchsformanalyse und systematischen gliederung von Teucrium L. I. Die Infloreszenzen una blüten. Flora, 168: 431467.

KÄSTNER, A. -1989- Übersicht zur systematischen Gliederung der Gattung Teucrium L. Biocosme Mesogéen, 6(1-2) 63-78. Nice.

KUNZE, H. -1989- Probleme der Infloreszenztypologie von W. Troll. Pl. Syst. Evol., 163, 187-199. 
MARESQUELLE, H.J. -1961- Sur la filiation des inflorescences. Bull. Soc. Bot. Fr., Memoires, p. 117-119.

MARESQUELLE, H.J. -1970. La thème évolutif des complexes d'inflorescences. Son aptitude à susciter des problèmes nơuveaux. Bull. Soc. Bot. Fr., 117: 1-4.

MEENA SHARMA \& V. SINGH, -1980- Trends of specialization in the inflorescence of Labiatae. J. Indian Bot. Soc., 59: 207-214.

MUS, M, ROSELLÓ, J.A. \& M. MAYOL. -1991De flora baleárica adnotaciones (9). Candollea, 46(1): 47-51.

NAVARRO, T. -1995-Revisión del género Teucrium L., sección Polium (Mill.) Schreb., en la Península Ibérica y Baleares. Acta Bot. Malacitana, 20: 173-265.

PUECH, S. -1984- Les Teucrium (labiées) de la Sect. Polium du bassin méditerranéen occidental (France et Péninsule Iberique). Naturalia Monsp., A5, 71 p.

RICKET, H.W. -1944- The classification of inflorescences. Bot. Rev., 10: 187-231.

RICKET, H.W. -1955. Materials for a dictionary of botanical terms. III. Inflorescences. Bull. Torr. Bot. Club, 82: 419-445.

SELL, Y. -1964- Les complexes inflorescentiels de quelques Acanthacées. Étude particulaire des phenomenes de condensation, de racémisation, d'homogeneisation et de troncature. Ann. Sci. Nat. Bot., 12 ser. 10: 225-300.

SELL, Y. -1969- les complexes inflorescentiels de quelques Acanthacées. Ann. Sci. Nat. Bot. 12 ser. 10: 225-300.

TROLL, W. -1950- Botanische Notizen. I. Abh. Akad. Wiss. Lit. Mainz, math-nat. Kl. Nr. 13.

TROLL, W. -1957-Practische einfuhrung un die planzenmorphologie zweiter teil die dlunhende planza. VEB Fischer, Jena.

TROLL, W. -1964/1969- Die infloreszenzen, Typologie und stellung im aufbau des vegetationskorpers, Vol. I, II. I. Jena.

WEBBERLING, F. -1963- Homologien im Infloreszenzbereich und ihr systematischer Wert. Ber. Dt. Bot. Ges. 76, 1. Gen. vers. H., 102-112.
WEBBERLING, F. -1989- Morphology of flowers and inflorescences. Cambridge University Press, Cambridge. 405 pp.

WILLKOMM, M. \& J. LANGE. -1868-Prodromus florae hispanicae seu synopsis methodica omnium plantarum in Hispania, II: 466-481. Stuttgartiae.

Aceptado para su publicación en Junio de 1995

Dirección de los autores. Departamento de Biología Vegetal. Facultad de Ciencias. Universidad de Málaga. Campus de Teatinos. Apdo. 59. 29080, Málaga. Fax (95) 2131944. 